Authors' pre-publication copy

Full citation:

Stewart, C and Pullen, E (2014) Monadic, material and mirroring: Female bodies in track athletics culture, International Review for the Sociology of Sport. DOI: 10.1177/10126902145449202

The online version can be found at:

http://irs.sagepub.com/content/early/2014/09/11/1012680214549202

\title{
Monadic, material and mirroring: Female bodies in track athletics culture
}

\author{
Carly Stewart \\ Emma Pullen
}

Correspondence:

Dr Carly Stewart, Cardiff Metropolitan University, UK

Email: cstewart@cardiffmet.ac.uk

Emma Pullen, Loughborough University, UK

Email: E.Pullen@lboro.ac.uk 


\title{
Monadic, Material and Mirroring:
}

\section{Female bodies in track athletics culture}

\begin{abstract}
This study explores how female track athletes experience and use their bodies within the contexts (social places) that form part of their everyday routines. Using ethnographic methods (focus groups, observations, self-directed photographic elicitation, and reflexive diary entries) the research focused on a training group of five semi elite female track athletes based in the U.K. in which, one researcher was a full participant. Arthur Frank's (1991) theory of the body is employed as an analytical lens to explore and illuminate the predominant types of body-usage manifest in their embodiments. The findings indicated that the athletes were predominantly mirroring bodies, with focus on appearance central to their experiences. Crucially these women desired more muscle in the mirroring process. Finally, attention is drawn to Frank's typology as a useful framework through which to contribute to some of the key issues related to women's experiences of their bodies in sport.
\end{abstract}

\section{Key words}

Body-usage, mirroring, female, athletics, muscle

\section{Introduction}

Women's bodies are at the centre of concerns (and debates) about female athletes and their sporting experiences (see Hall, 1996; Hargreaves, 1993; Markula, 1995; Theberge, 2000; Cole, 1993), where gender is a structuring principle for understanding how people experience themselves in and as bodies (Woodward, 2011). One of the particular concerns many sportswomen have with their bodies is the development of a muscularity not immediately associated with orthodox femininity. On the contrary, the common sense notion of the powerful athletic sporting body remains a key definer of masculinity in Western culture (Connell, 2005), and accordingly anxieties exist around just how much muscle women should sculpt in sporting, exercise and physical culture contexts. Studies have highlighted the paradoxical relationships that women have with their bodies in relation to athleticism across these contexts (Cox and Thompson, 2001; George, 2005; Krane, Choi, Baird et al. 2004), however relatively little work has been done on female track and field athletes (Mosewich et al, 2009). Indeed, unpacking the various differences between event-specific 'body projects' (Shilling, 2012) within track and field remains largely unstudied. We contend that this is a sport of particular interest where a continuum of athletic female bodies (George, 2005) are visibly displayed through a range of events, and arguably some of these represent an emerging cultural female athletic-ideal. Given this, and calls for more work to be done to explore embodied practices and lived experiences in sport (Allen-Collinson, 2009; AllenCollinson and Hockey, 2007), in this article we use Frank's (1991) theory of the body (a 
typology of body usage) to explore how a group of female track athletes experience and use their muscular bodies.

\section{Women, bodies and sporting experience}

The cultural meanings assigned to bodies are crucial to the experiences of sporting females. As Cox and Thompson (2001: 18) explain, 'women who use their bodies to play sport, an institution largely constructed by men, often experience contradictions, ambiguities and conflicts'. It is against this backdrop that physically active females experience paradoxical relationships with their bodies and these are widely documented across a range of sports, exercise and physical culture contexts. For example, Krane et al (2004) explore how collegiate female athletes negotiate femininity and athleticism in the U.S. which include distance runners, track athletes, soccer, tennis, rugby, ice hockey, volleyball, and hockey players. The athletes in their study expressed being proud of their strong developed bodies, but also of a concern at 'becoming too muscular' because it detracted from femininity and the cultural body ideal (Krane et al, 2004: 320). Similar ideas of a 'glass ceiling effect' (Dworkin, 2001) were found by George (2005) in a study on elite collegiate soccer players. George (2005) reports that whilst the muscular physique once largely unacceptable is, to some extent, desired, an upper limit as to what amount of muscle is acceptable for women to possess operates. Importantly, like the athletes in Krane et al's (2004) study, the soccer players embraced the performance potential of their muscular bodies. Similarly, in New Zealand, Cox and Thompson (2001) also found that female soccer players experience their bodies as having physical presence whilst playing their sport, but that this presence is mediated by a desire to be seen as feminine within the discourses of sport, gender and heterosexuality. Importantly they pay attention to a multiplicity of corporeal tensions experienced on the soccer field and in social settings which require constituting themselves accordingly through gendered practices as associated with dress and hair, for example. Comparable tensions are found in other sporting and physical culture contexts which include boxing (Mennesson, 2000; Paradis, 2012) and bodybuilding (Wesley, 2001; Roussel and Griffet, 2009; Roussel, Griffet and Duret, 2003) where hyper-muscularity is a means to feeling empowered and femininity is often emphasised in order to make muscle 'safe'.

In boxing, much like athletics, musculature is visibly on display where the surface of the body matters and is related to ideas about performance. Paradis (2012:90) explains that sport subfields often have implicit blueprints which define 'the ideal-typical body' of the ideal practitioner. These athletic 'specimens' are afforded and command more attention from everyone, ranking higher on the social hierarchy of that field because their bodily appearance directly maps onto beliefs about ability. In the case of boxing, these are described as being muscular, lean, dark and fierce male bodies (Paradis, 2012). In an ethnography of her experiences as a full participant, using Bourdieu's theory of practice, Paradis (2012) pays attention to her own bodily capital, in particular readings of it that impact micro-level interactions and hierarchies at a boxing gym. Bodily aesthetics and gender were central to her experiences. For example, she reports that her bodily traits served her well in a boxing context; despite her body being read as 'woman' it also signalled 'fighter' because of her visible muscularity. However, the same muscularity takes on a different meaning in the social 
setting of a dance club, where she moves from 'fish in water' to 'fish out of water', illustrating that the female athlete's relationship to her body is fundamentally relational, contingent on schemes of perception and appreciation of bodily traits and symbolic capital in the field. Importantly, Paradis' (2012) work argues that the body centrally matters for social interactions and participates in the legitimation of social actors in social space, providing insight into contexts and bodily hierarchies.

With the exception of Mosewich, Vangool, Kowalasi and McHugh (2009), female track and field athletes have received little attention from academics in relation to these issues as a specific sporting group. Mosewich et al (2009) focus upon the ways in which eight athletes from a range of events (including sprinters, long distance runners and pole vault) described their physiques and understood their meanings of muscularity. Importantly they found that these meanings vary amongst athletes and with context; muscle is desired for performance by some, but also for appearance concerns in certain contexts, by others. These findings are consistent with those in the aforementioned research in relation to difficulties in retaining femininity and building muscle. Interestingly the sprinters in Mosewich et al's (2009) study desired a greater amount of muscle, but the appearance of muscle (tone) played an important role in what constitutes an 'ideal' physique. Additionally they found that appearance and performance were difficult to separate in relation to desired muscularity goals, and that a culture of comparison amongst the athletes was present; of wanting to look like each other and other elite athletes they knew. Despite outsiders viewing them as having athletic 'ideal' bodies, issues of struggle with self-acceptance were prevalent with photo elicitation illustrating self-perceptions and body-self-other relationships as an on-going project for them.

Mosewich et al's (2009) study provides an insight into this under researched sporting group and some purchase on how track and field athletes perceive their athleticism in relation to cultural frames. Despite this, questions remain concerning how their bodies are used and lived in everyday interactions and contexts. This said track and field female athletes as a sporting group warrant further exploration in relation to these questions. We suggest that the group of sportswomen presented in this study are an interesting case in a sport where a continuum of body ideals referred to by George (2005) exists, and where intercultural differences inscribed onto bodies are displayed and noticed more so than in other sports. For example, one athlete explains: 'You can really tell what people do...you can point out the distance runners, compared to the high jumpers, compared to the throwers, or whatever' (Mosewich et al, 2009: 105). In relation to this, bodies are afforded differential value in comparison to an emerging ultra-fit toned athletic-ideal for female athletes (Homan, 2010) ${ }^{1}$. Those who compete over distances of 200, 400 and 800m, may construct powerful bodies that are highly muscular with tone (the appearance of no or little fat) but within the confines (or on the boarders) of what might considered acceptable femininity when compared to other distances or events. For instance, 100m runners who develop and display significantly more muscle mass may cross the boundaries of unacceptable femininity for some. This study seeks to build upon and address gaps in the work of Mosewich et al (2009) to understand how a small group of track athletes who compete over varying distances construct, maintain and experience their bodies whilst occupying a position on a continuum of ideals. 


\section{Theoretical Perspective}

Whilst Mosewich et al's (2009) findings certainly attest that the athletic body is a chronic corporeal presence in the lives of female track and field athletes, the scope of their analysis is deliberately limited to perceptions of muscularity and athleticism, and does not explore the relationship between the body and the social contexts which produce embodied action. Like Paradis (2012) and in line with Shilling (2012) we sought to go beyond approaches which neglect the emergent properties of embodied subjects. This said our theoretical perspective is one where materiality matters and the interactions between embodied subjects and social phenomena are focused upon, taking seriously the existence of the body as a corporeal phenomenon which itself affects how people experience their bodies (Frank, 1991). Thus our position on the body is that of a 'multidimensional phenomenon' with attention paid to dimensions of its 'receptive and productive capacities' in a more fully embodied perspective (Shilling, 2012:241). In this sense we recognise that embodied subjects are not just locations for the transmission of social classification, but that they possess physical and reflective capacities, and are actively generative of social relations and human knowledge.

Understanding how these women not only describe, but use and live their bodies in relation to each other is a key concern for us. It is against this backdrop that Frank's $(1991 ; 1995)$ work provides a useful theoretical framework to illuminate how a group of female sprinters experience and use their athletic bodies.

\section{Frank’s Typology of Body Usage}

The work of Frank (1991) has been usefully employed within a sporting and physical culture context to illuminate the construction of 'ideal' body types or 'typical' styles of usage by individuals (Sparkes, 2004; Sparkes, Batey and Brown, 2005; Stewart, Smith and Sparkes, 2011). For Frank, the body is a starting point, and he takes seriously the existence of the corporeal fleshy body which itself affects how people experience their bodies. More specifically, he argues for the body as 'both medium and outcome of social body techniques' (Frank, 1991: 48). The body then is not a functional problem for society (looking down) but an action problem for itself ('body up' approach), proceeding from a phenomenological orientation. He isolates four questions or 'action problems' which the body must ask itself as it undertakes action in relation to some object. These problems are of control, desire, selfrelatedness and other-relatedness. As bodies respond to these four action problems, typical and discrete styles of body usage emerge. These are: the disciplined body, the mirroring body, the dominating body and the communicative body. Here we limit ourselves to an overview of the four action problems, and just two styles of body usage in detail-the disciplined and mirroring bodies_-produced as they respond to the four action problems. These bodies are of specific interest to this study.

The first action problem is of control. In any given situation the body must ask itself how predictable its performance will be: Can I reliably predict how my body will function? Can I control it's functioning? According to Frank (1991: 51) 'bodies always align themselves somewhere on the continuum between god-like assurance and the embarrassment of a Freudian slip'. The body of a gymnast, for example, may lie at the end where predictability is 
at its highest expression and a body subject to extraneous uncontrollable forces at the other. The second problem is of desire, and whether it is lacking or producing: What do I want, and how is desire expressed for my body, and through my body? Desire is the quality of always wanting more, and yet of being unfulfilled. Consumer bodies, for example, fit this description of never being satisfied. The third problem is of the body's relation to others, concerned with how the shared condition of being bodies becomes a basis of empathetic relations among living beings: What is my relationship as a body to other persons who are also bodies? Does the body relate to itself as monadic and closed in upon itself, or dyadic, and existing in relation to others? The monadic body understands itself as existentially separate and alone, for example, it bears its own pain. In contrast the dyadic body understands itself as a medium through which other bodies are connected, for example, as part of a 'brotherhood of those who bear the mark of pain' (Schweitzer cited in Frank, 1995: 35). The fourth problem is of self-relatedness (Frank 1991) or body-relatedness (referred to later in Frank, 1995): Do I have a body or am I a body? The disassociated individual may see the body metaphorically as a garment which does not have serious implications for who the individual is. On the other hand the associated individual may perceive clothing as covering and revealing at the same time, saying something of the individual.

In response, four ideal types of body usage emerge through their respective media of activity used to resolve these action problems in different ways. The disciplined body defines itself through the medium of regimentation and its most important action problems are those of control. So long as a regimen is followed, the body can believe itself to be predictable. The responses or 'tasks' required of the disciplined body to the action questions within a sporting context are amplified by Sparkes (2004) in his analysis of cyclist, Lance Armstrong's autobiography. Sparkes (2004) draws attention to Armstrong's twice daily intense training regimes in which he seeks to be predictable through precise quantification, self-monitoring and subjected to the constant surveillance of others. Further, the elective affinities of the disciplined body to respond to tasks posed are evident in Armstrong's reaction to cancer diagnosis. Suffering crisis in the lost control of his body, Armstrong seeks to restore predictability by means of therapeutic regimentation. The body is monadic, an 'it' to be treated. The work of Stewart, Smith and Sparkes (2011) supports this analysis, highlighting mechanistic metaphors which reveal the disciplined body to be understood (and related to) in terms of a machine.

In line with more contemporary regimens, 'there are those for whom discipline of the body becomes its care' (Frank, 1991: 61). The mirroring body defines itself in acts of consumption that enhance the body in an 'endless assimilation of the world's objects to one's own body, and of one's own body to the world's objects' (Frank, 1991: 62). In this sense it attempts to recreate the body in the image of other more desirable bodies, seeking predictability of appearance and conformity to internalised images (often superficial). Where the disciplined body fears disruption to its work routine, the mirroring body fears disfigurement. It is also monadic, but produces desire (always wanting more) and is fully associated with its own surface; the visual is primary. This body is most commonly associated with consumer culture. An example is the instrumental shopper in pursuit of artificially-framed styles of life, void of 
relating sensuality to communication with others. In a physical culture context, Sparkes, Batey and Brown (2005) suggest that body builders provide an excellent example of the mirroring body in action. Highly refined diets and training regimes promote optimal conditions for muscle development where the visual is primary. Drawing on the previous work of Brown (1999) they note how 'bodybuilders desire and use the gaze of others in relation to their hyper-muscularity, along with their own constant self-scrutiny, to construct and confirm their identities as their flesh is transformed' (Sparkes et al, 2005: 132). This said body builders are acutely aware that they are their bodies.

Importantly, Frank (1991) emphasises that his model is not as neat as it may appear. He refers to the specific body styles as 'four puppets that dance, and sometimes dangle, at the theoretical ends of these four continua' (Frank, 1995: 40), that these ideal types are not meant to encompass all possible types of usage but serve as heuristic guides, through which, bodily behaviour can be understood:

To write of 'the disciplined body' is to suggest a typical style of how the body is experienced and deployed. Of course empirical bodies will not stay long with one type of usage; again the truth is a mess. But the objective is to generate heuristic guides through which to order empirical behaviours and understand something of their flips and relations (Frank, 1991: 53)

This said the bodies in this study are discussed on a level of empirical description, as ideal types whose specifications provide some interpretive understanding of how bodies exist at different moments of their being.

\section{Methods}

This study is an ethnographic study (Brewer, 2000) of a training group that consists of female track athletes competing across the events of $200 \mathrm{~m}, 400 \mathrm{~m}$ and $800 \mathrm{~m}$. These athletes (five in total) are aged between 21-26 years and can be described as semi-elite based upon their level of representation and time invested in their athletic careers. Their rankings range from 8-30 in the UK with three having represented their respective countries in International domestic competition. All invest in a significant amount of training required to compete in major National competitions. In terms of assigned social categories, all are of white ethnic origin, middle class, and all identify as heterosexual with the exception of the athlete-researcher (EP). This admission becomes important where the researcher occupies a unique position within the group as full participant. As Bruner (1997) and Davies (2002) note, the personal relationships and similar cultural resources shared by the participants is an important part of the observational data. In embodying an alternative identity to that of heterosexual track athlete and due to rapport with the group, the athlete-researcher was able to collect rich data, sometimes of an intimate nature ${ }^{2}$. Her positionality, embodiment, and the pre-conceptualised understandings of the athletic subculture interacted with one another in a reflexive manner as noted elsewhere (see Crossley, 2006). Further, elements of this study may be read as 'analytic autoethnography', described by Anderson (2006). Sharing a commitment to features of traditional symbolic interactionist ethnography, it involves complete membership, sustained 
reflexive attention to one's position in the field (of its discourse and relations), textual visibility of the self in ethnographic narratives, and a commitment to an analytic agenda. Importantly, Anderson (2006) notes that goal of self-understanding is one which lies at the intersection of biography and society;

'understanding our personal lives, identities, and feelings as deeply connected to and in large part constituted by - and in turn helping to constitute - the sociocultural contexts in which we live' (Anderson, 2006: 390).

To clarify, data were collected via ethnographic field notes (Brewer, 2000), focus groups, photography and self-reflexive notes over a focused period of 6 months (though, for EP, the reflexive process involving this group and her own biography extend to a period of approximately 4 years). Photography as part of data collection was used. It was directed by both participant and researcher, and subsequently used for elicitation purposes in the focus groups. A digital camera was made available during the period of research, placed in a position where it could be accessed easily by the group. Participants were invited to take pictures at their own will. The photos produced were predominantly of times when athletes' bodies were on display, for example whilst lifting weights in the gym where muscles were revealed and little clothing was worn. Having been informed of the purpose of the study participants exhibited excitement at the opportunity to use photography in this way. This becomes an important admission and adds weight to our analyses where a focus on appearance of bodies was very much driven by participants. Further, as Harper notes (2002) using photographs in the interview setting acts as a stimulus for conversation, reflection, and engagement with the sensorium. Accordingly, we sought to encourage focused reflexivity to their bodies and movements captured in the photos, and to provoke narration of experience (Harrison, 2004). Photographs instinctively drove the content and questions within the focus groups. An example of the types of questions asked by the researcher include: 'Why did you take this picture?' 'Tell me about this picture' and 'How do you feel about this picture?' More often than not focus groups would begin with photos being displayed and conversations about bodies would emerge organically, without effort, and with a focus on the appearance of bodies taking priority. Finally, in line with Frank’s $(1991 ; 1995)$ work which takes seriously the fleshy, corporeal existence of the body as central to how people experience themselves, this study is phenomenologically sensitive both in its theoretical approach, and as an inherent part of the research design which pays attention to the sensory; sound, smell, touch and taste (Sparkes, 2009).

\section{A spatial tour}

To understand and illuminate some of Franks' (1991) specific modes and shifts in body usage brought about though the contextual answering of action problems, we chose three social spaces to focus our attention based upon the group's daily routines. In doing so, we recognise the importance of places in the athletes' lives as socially and culturally constituted social spaces through which they move, filled with objects, representations and above all meanings (Soja, 1996). Data collection was focused across three specific social places that the group frequently occupied. These were: the athletics centre, the weights room and the coffee shop. 
All three places consist of intelligible or meaningful material arrangements which are tied to the performance of particular activities within the University sports focused campus. Sporting bodies are an integral part of all three. A brief overview of each is provided to set the scene:

The athletics centre: Contains indoor and outdoor tracks, both are used by the group on four days of the week for a period of 3 hours in any one day. Primarily this centre is used by track and field athletes in training but it serves as a space for sports lectures and as an occasional training facility for other sports groups (e.g. trampoline gymnastics and rugby).

The weights room: Distinctively designed for track and field athletes and contains predominantly free weights, lifting platforms, racks and bars. It is situated within the athletics centre and is a relatively small bounded space set back from the main area. The groups use the gym on the remaining 2 days of their 6 day training week, again for a period of 3 hours in one day.

The coffee shop: Significantly different to the aforementioned spaces, both in terms of physical structure and how it is used by the group. The group congregate here after training sessions and select a seated area to occupy. The space is also inhabited by other sports people, university students and staff. The group spends up to an hour and a half in here in any one day.

In what follows, findings are presented and should be read as 'modified' with EP's own voice and authorial positions as participant, observer and researcher central to narrative practice in an interpolation of vignettes, researcher diary inserts, interview extracts and photographs.

\section{Findings}

\section{Disciplined and mirroring body usage}

There was a strong presence of disciplined and mirroring body types. During observational periods athletes were engaged in either focused physical conditioning of the body or in interactions consistently framed around 'body talk'. Whilst both disciplined and mirroring body usage was observed, the mirroring body took centre stage in focus groups and emerged as the more predominant mode of being, reflected in the presentation of findings that follow. The entwining of these specific body types are illuminated through their responses to the contextual action problems described by Frank $(1991 ; 1995)$ and are illustrated below.

\section{Action Problems of Other relatedness and control:}

\section{Monadic and predictable bodies}

The contextual action problem of other-relatedness - what is my relationship, as a body, to other persons who are also bodies? - remains a key concern for the mirroring and disciplined body types. The monadic body is one response to this action problem, reflected in understanding itself as existentially separate and alone. It may be of no surprise that athletics culture encourages monadic bodies, articulating well with an emphasis on individual achievement. For example, it was observed that whilst these athletes train in close proximity 
to one other, meaningful contact can sometimes be difficult to discern. Friendships are formed in these spaces but our observations suggest that contact is often minimal and transitory during training. More often than not the athletes are monadic in their embodiment. In the case of the disciplined body, this is observed where athletic bodies are pitted against each other in both training drills and racing. One reflexive diary extract recalls how movement is orchestrated within the indoor athletics centre, with discrete spaces (circuit stations and individual running lanes) designed for individual bodies that are not to be shared:

We have just finished a session outside and as we get ready to begin the next session which is circuit training, we strip down to our crop tops and sports bras in anticipation. I look around at our group. Including the boys, there are approximately 8 to 12 of us and we are visible to others by our bodies on show. As we move around the athletics centre, a large open space occupied by a range of bodies, our group is discernible by our particular body shapes and movements. I have begun to monitor how the girls, myself included, move as we migrate around the vicinity. During the circuits, voices reverberate with the coach shouting orders at a pitch that subjugates the noise of the group. I hear Rachael shout at one of the boys as they are slow moving off one of the stations, 'Get out my way, now!' She shouts, and Tom moves quickly off to the next station. I make a mental note to myself to record the aggressive manner she uses, or perhaps importantly, Tom responding without argument.

The pattern of individual relating is reflected in the use of this space, and further illustrations lie in the observation that athletes often relate individually, not collectively, to the group coach.

In the case of the mirroring body, responses to problems of other-relatedness are observed where other bodies in the group become benchmarks (objects) not just for performance but for appearance also. Despite awareness of their shared corporeality, the group often engaged in exchanges which served to aid in constructing self in the image of another idealised body. More often than not these exchanges were viewed as monadic and not dyadic. That is notably to each other rather than for each other. In this sense others are situated as 'instrumental allies' (Frank, 1995: 44) or as an 'audience' with specific performance expectations. In the following comments Rachael relates to other female athletes as bodies which are objects to be evaluated, devoid of humanness in the strictest sense, and are used instrumentally for the reflexive construction of self:

Rachael: 'For example, if you were to display the girls, like the girls in our group, and you just put their torso and cut their heads off from their abs I could probably point out who was who -- and their bums'

Rachael: 'I think as well, if one of the girls gives you a compliment about your physique or whatever in the gym then I will go out my way a little bit extra next time to get the compliment and try to reinforce the, you know, 'you look good"'

Compliments about the athletes' bodies given by other females are of far greater worth and induce a greater sense of pleasure than those made by male athletes, reflecting further the monadic and instrumental use of other females in these contexts. Throughout the research, very little interaction (tactile or verbal) concerning physical bodies was made between male 
and female athletes. Esther explains that other female athletes have something of an expert gaze when compared to male athletes:

Esther: 'I would appreciate if you [EP] said to me "oh like you're looking good or you're not looking so good", I would appreciate that because it comes from someone that knows'

Esther: 'I don't like looking at guys really but then you look at girls and you think that's really impressive you look really good, the boys don't really know what they are talking about and I don't think they would even say it anyway'

When it comes to action problems of control—can I reliably predict how my body will function? - both the disciplined and mirroring bodies align with a shared response of expressing a high level of predictability in performance and appearance respectively. That these athletes seek predictability of performance is overtly evident in their intense training regimes and repetitive drills. There are moments where the drills must be completed in the single minded pursuit of training above all else, illuminating the disciplined body. However, at other times the predictability of appearance is also evident in the concerns and time given to regimes of bodily care needed to look good, illuminating the mirroring body. For example, selecting the 'right' training kit $^{3}$ is important in achieving this goal where bodies are on display in training, and even more so in racing. EP recalls a specific moment at a competition where control of appearance is clearly highlighted:

Sudden movements divert my thoughts once again as Lucy swings her legs onto the bench and begins to apply fake tan. Her acknowledgment of her fair hair and fair skin attributes is made as she quickly laughs and begins the process of neat application. I ask her 'why?', 'tanned skin looks more muscular, it brings out the tone', she replies. She is racing in about 90 minutes and explains that she needs to get it finished and dry before she starts to warm up. I can smell the aroma of the dark sticky mixture that is sitting on her skin, it has always smelled to me like biscuits and sunscreen mixed together, and as it hits my nostrils the smell organises my thoughts back to the weekends of athletics championships where I have shared a room with her. I realise this is the first time she has undergone her preparation in such an open space but this is the women's athletics league and female bodies are everywhere.

In another example, Rachael clearly refers to training as a regime needed specifically for controlling appearance. She also identifies the context of racing as a specific moment where this matters most:

Rachael: 'Especially when you are on the big screen and it was like, "I need to make sure I look good because the whole stadium is going to be seeing me"..... I think you know when people go on a night out but don't train for anything that's their time to look at their best but when we train and we put so much time into our performance that it's the same concept, that's our time to look good, not just performance wise but going out with crop tops and knickers on'

\section{Action problems of Desire and Self-relatedness:}

\section{Desiring and compulsively associated bodies}


As illustrated, disciplined and mirroring bodies exhibit shared responses to action problems of other-relatedness and control. They differ, however, in their responses to action problems of desire and self-relatedness. The contextual action problem of self-relatedness-Do I have a body, or am I a body? — elicits responses that reflect bodies as either 'associated', and connected with their surface, or 'disassociated' and not connected with their surface. The disciplined body, for example, is disassociated from its surface and does not care about its appearance in instrumental pursuits (e.g. during training drills). The mirroring body on the other hand is highly associated with its surface which has implications for who the individual is. The associated body emerged to add further support for the presence of the mirroring body as the predominant mode of body usage aside of training and racing. Examples of high body association are seen threaded throughout the entire data set that precedes this section and that which follows, where the visual (surface of the body) is primary and concerns about appearance are paramount (e.g. selecting the 'right' kit, concerns about being seen on the big screen at competitions and applying fake tan).

Further, related to appearance concerns, is the contextual action problem of Desire- - what do I want, and how is desire expressed for my body, and through my body? Again this calls upon a specific response by the mirroring body-it expresses desire of wanting and needing more. Data reveals that a body associated with an athletic-ideal is what's desired. The athletes offer very specific insights into what this might look like which include references to each other's bodies and those of well-known elite athletes, such as British heptathlete Jessica Ennis-Hill:

Rachael: 'It's like I want to look like, like I want a pair of Jessica Ennis' abs, it's a goal, I will have abs like that, I will have a bum like she has, everything is always working towards something which is I guess what we train for as well'

Expressing desire, for, with and through bodies as a corporeal demand and need is evident throughout. The group are particularly eager to discuss each other's bodies and the photographs in focus groups. Sometimes these discussions transpire in training contexts illustrated in the reflexive diary extract below:

Esther strode over to the gym in her work uniform and grabbed at my arms, 'look at the definition here', Tiffany looked up from here exercise and commented, 'I know it's amazing, can you see I'm starting to get veins in my shoulders, Emma, now I'm doing more weightlifting? I think I want to be a weightlifter', 'Yeah you look good' I reply. Esther continues, 'are we doing another focus group soon with the photos? You should have had one of Tilly's bum it's amazing, I would of loved that', Tilly laughs and I comment, 'I could get one for the next time'.

The coffee shop was used as a place for the group to meet and discuss the photographs, and during focus group time became a place where desire was openly and elaborately expressed. This said the use of photography induced very little, if any, shyness or modesty at all amongst the group. Instead, engaging with the photos became a further means to judging, comparing 
and internalising other bodies. Figure 1.0 and the conversation that ensued illustrate moments of expressing desire, and of consuming other bodies:

(Figure one)

Esther: 'I would have your bum-- and your hamstrings-- and I love how you have so little fat there, its nice and defined and I remember my bum like that and it's nice to touch so I would have your bum and your hamstrings and the hip shape'

Importantly, although the mirroring body produces desire, its desire is monadic; 'what the mirroring body wants, it wants for itself' (Frank, 1995: 44). Frank (1991: 63) draws upon the concept of window shopping to explain how objects need not be purchased because they have already been consumed in gaze. He writes, 'For the ultimate mirroring body, it is simply enough to walk through the shopping malls, to see what is there, perhaps to 'try on some things'. According to Frank (1991), what counts is the endless producing and reproducing of desire, highlighted in the following example where other bodies are consumed through gaze:

Esther: 'Because I would be like, "right I want her shoulders, her abs, her arse, her legs" and I used to pick out what was realistic for my body shape, and the size and height and everything, and what areas need to be stronger and be as good as these other girls and better'

In relation to the amount of muscle desired, interestingly, all expressed wanting more; concerns about becoming too muscular were not aired at all. On the contrary, Esther expresses a wish to become a weightlifter. These findings are, in this sense, different to those in previous studies where an 'upper limit' and concerns about become too muscular have been expressed (e.g. Dworkin, 2001; George, 2005). Desiring bodies are illustrated further still where EP records hearing contemporary metaphors of desire used often during their interactions as a group. Importantly Kirmayer (1992) notes that while metaphors may be used without awareness or concern, the body partly makes itself presence known through metaphors grounded in social interaction and bodily experience. In the following examples, the presence of the metaphor "desire is heat" is illuminated through the use of the term 'hot', employed to express (often sexual) attraction for a very attractive woman:

Izzy and Rachael sit down next to me and start to pick at the bag of sweets, ready to watch Lucy race. The $400 \mathrm{~m}$ hurdles are ripe with similarly defined bodies and as I watch the bodies prepare their starting blocks in the lane, Rachael comments on the athlete nearest to us. 'How does her bum look so hot in race briefs'? I hear these comments often from the girls, as I start to become aware of the open dialogue that exists between the group as we see other girls racing or training. (...)

Esther: 'Even in training, I have always been looking and thinking she looks hot, you know when she was in uni and doing the physio, and I remember she got to a point when she was so lean and she was so ripped and I have never seen her looked so ripped, and I was thinking oh my god, amazing and I remember finding myself following her around the track in complete awe and my coach was like concentrate and stop looking at other people' 
We had very little sense that this desire was dyadic and cooperative- - they did not actually want to have sexual relations with each other-but instead use metaphors as a way of expressing instrumental desire, of wanting for self. Importantly, metaphors are culturally contingent language devices, and their availability for use tells us something of the culture from which they are derived (Edwards, 1988). The use of sexual metaphor by females in relation to other females here is interesting, and provides a different picture of sexualised sports talk to that painted by the literature so far where sexual metaphors are used by sportsmen to objectify women (see Curry, 1991).

The analysis so far indicates the presence of the mirroring body as a predominant mode of usage, with the appearance of the body paramount to these athletes' experiences. However, we have also illustrated the presence of the disciplined body in training contexts, and suggest that body usage switches between these two modes depending on the context. In a continual switching between these modes of embodiment, the findings provide support for, and shed further light on, Mosewich et al's (2009: 105) acknowledgement that there appears to be somewhat of a 'blurred line' between appearance and performance for female track and field athletes. Like Mosewich et al (2009) our findings also suggest that appearance and performance are interconnected and often difficult to separate, particularly in relation to muscularity. The athletes in this study acknowledge the function of muscularity and its benefits to performance but simultaneously recognise the effect of training on their appearance. The athletes' complex negotiation of the relationship between performance and appearance is evident in the following comments. In the first, Esther relates looking good as a requirement to feeling good when racing:

Esther: 'When you go out on the track and you feel, "oh my god I am looking good today", you are so much more confident in your actual performance'

In the second, the perplexed nature of considering what's more important to the body projects they undertake - performance or appearance — is evident and the two are clearly conflated in her comments:

Esther: 'It's like you know you have to have fat to stay healthy but we don't care about that we want to look good and looking good and performing good, when you look at the anorexic side of things that is just a completely different mind set where we want to do it more for performance reasons---We want to get ripped and show our muscles off'

Given the presence of the mirroring body which is so central to the athletes' everyday experiences there is cause to consider that appearance concerns are given at least equal weighting, if not more, than performance concerns for some of these athletes.

\section{Uncomfortable transitions and the importance of place}

As Frank (1991) asserts, problems of embodiment arise from the social context in which they take place. Each body problem is a problem of action: to act requires a working resolution to the problem when engaging in social inter/action (always within a social context). A change in social context then may bring about a change in body problems faced, and therefore in 
responses. So far we have illuminated Frank's (1991) disciplined and mirroring body usage as produced comfortably by the athletes in response to body problems encountered in training places. We have suggested that mirroring and disciplined bodies are in continual flux, responding to resolve action problems that mediate between athletic performance and looking good. A focus on the places inhabited by the athletes provides further support for our analysis of these body ideals as central to the athlete's experiences. For example, as the group reflects upon moving from the athletics centre to the coffee shop their narratives relay a shift in embodiment (from equilibrium of comfort to discomfort) despite still being in close proximity as a group. EP's reflections set the familiar scene where this shift often occurs:

It is busy in here today; a high pitch hum oscillates in pulsations to the excited conversations and laughing of a group of dance students sat on the sofas next to us. The general busy movement of a lunchtime rush in the coffee shop. Between the deeper notes of male voices, higher octaves of interactions flow between females. There are also sounds of milk being steamed and periodic banging and grinding of the coffee machine. The group move into a space between the dancers and the wall-far too small to accommodate us-as we squeeze together, pulling chairs closer from empty tables and invisibly shifting the spatial limits outwards. I feel that we have started to encroach upon the dancers table. Conversations turn to training. There is a considerably less 'body-appearance-centred' conversation now that we have left the athletics centre. Talk is on times and championships and training sessions. One of the girls appears at the table with a protein shake, 'is that all you're having for lunch?' coach asks. She replies, 'yeah trying not to get over 62kg'. Is this still the presence of a mirroring body? Away from the gym in the coffee shop, with bodies covered up, anthropometric lives are lived out. What I have come to notice as the group occupies the coffee shop is the change in dialogue that accompanies this space. Body talk is continuous but wrapped up in the chronologies of training orientated talk. The flagrant intimacy of bodies is averted by the covering of them in tracksuits and of bodies living out converging narratives in a different context.

Similarly, the group report how their talk differs in this particular place. They reflect on a complete absence of sexualised language expressed through desire when talking to one another outside of the research context. Rachael and Esther explain how the coffee shop silences sexualised dialogue abruptly:

Rachael: 'I think the way we talk to each other is completely different when we move from the athletics centre to the coffee shop' (...) 'Yeah I become very cocky in the gym, not outspokenly but I mean when I walk and talk, you know a bit of swag but as soon as I leave that goes'

Further, changing places (social context) is an uncomfortable move that is felt at a bodily level. For mirroring and disciplined bodies, both connected through their concern with action problems of control and other-relatedness, having high levels of predictability and being monadic is difficult in a new environment which presents contingency in bodily routines. This sense of not being in control is feared by the athletes and the coffee shop presents a social context where their sense of control is diminished. Esther reflects upon the shift and the embodied discomfort which ensues: 
Esther: 'I'm very comfortable in places like the athletics centre because I'm controlling and I control my training and that's how I behave and that's how I move and I am comfortable with that in the athletics centre, but I go somewhere like the coffee shop and I have to step out of that and I find it really hard---I feel stressed sometimes because it's such an uncomfortable situation'

These findings not only illuminate the presence of mirroring and disciplined bodies as the predominant mode of being, but also raise questions about being bodies and the distinctive problems of continuing to be the same sorts of bodies they have been across everyday social contexts.

\section{Discussion and reflections}

Despite being part of a culture where a range of muscular bodies are on display, female track athletes have received relatively little attention from academics in relation to issues of embodiment. Whilst Mosewich et al (2009) offer a preliminary insight into track and field culture and the way that female athletes describe and understand their muscularity in relation to cultural frames, they tell us less about the relationships between the body and social context which produce embodied action. Building upon this work, this article has paid serious attention to context than previously researched, as a way to understand the embodiments of a small group of female track athletes. Frank's (1991) typology has been employed to understand and illuminate specific (ideal) styles of body usage manifest among the group. In doing so, the potential of Frank's work to 'incorporate a view of the body as a corporeal phenomenon' is painted (Shilling, 2012: 102) and we have a better understanding of how bodies experience 'tasks as imposed by a system' (Frank 1991: 48). In other words, the body (its materiality) matters, and is a starting point-a problem for itself in understanding the lived experiences of these track athletes.

Indeed, as has been suggested, the disciplined and mirroring body types are illuminated in the data via their responses to the four contextual action problems, with the mirroring body emerging as the predominant mode of usage in everyday training places. Mirroring bodies are the kinds of bodies that constantly seek to recreate the image other bodies, more attractive bodies - in this case, more muscular and with more tone. The visual is primary and mirroring bodies are judged by appearance. Importantly 'in the world of the mirroring body, projection and introjection take place in seamless reciprocity’ (Frank 1991: 62) in an endless producing and reproducing of desire. We suggest that, in Frank's (1991) terms, the female track athletes provide an excellent example of the mirroring body in action. The responses to each of Frank's action problems raise some important points for discussion, shedding further light on the body of literature which currently exists around the lived embodiments of female sportswomen.

A first point for discussion concerns desire. Frank (1991) recognises that bringing women into sociological theory begins with recognition of women's differential conditions of embodiment. We cannot ignore the gendered dimension of desire as it relates to social and cultural ideas of what is or should be desired (and thus mirrored). As previously noted, being 
too muscular (bulky) for sportswomen negates, or detracts from, the feminine cultural body ideal (e.g. see Krane et al, 2004) but there is some sense that an 'athletic-ideal' (Homan, 2010) is changing to include pronounced musculature for sportswomen (George, 2005). Our findings certainly attest to desiring some form of athletic body ideal (as opposed to a thin model-like body ideal) similar to that described by the track and field athletes elsewhere (Krane et al, 2004; Mosewich et al, 2009). However, the athletes in this study not only expressed wanting more muscle, but they seemed unconcerned with the idea that they could become too muscular. The sprinters in Mosewich et al's (2009) study also expressed similar desires of wanting a greater amount of muscle, but specified that it must be toned (as opposed to bulky). Our findings are somewhat different those which have found 'upper limits' of musculature (e.g. Dworkin, 2001; George, 2005). In the aforementioned studies participants are asked to directly describe the tensions they experience with their bodies and muscle unlike this study where expressions of desire for muscle emerge through bodies within contextual practice and interaction. This said we contribute to existing work on female athletes and their bodies, providing a different theoretical framework from which to view in context the relationships these track athletes have with their bodies.

Further, we consider the athlete-researcher's role as full participant and the close relationship she has with the group to facilitate open discussion about desire. The group were comfortable, fully engaged and excited at the opportunity to indulge openly in the satisfaction they get from their bodies in the context of their lives which, might be reasons for a focus on the satisfaction that they have with their bodies as opposed to discomfort. This said studies have tended to focus upon the problematic relationships sportswomen have with their bodies and less upon the enjoyment, pleasures and satisfaction they get-something this study contributes. It is worth noting that these athletes were clearly in great physical condition (muscular with low body fat) and therefore may benefit from aligning closely to a female athletic-ideal, affording them comfortable space to enjoy the muscles they have. Further still, it is important to note that as athletic bodies these athletes may offer considerable draw to others pursuing an athletic ideal, as they do to each other. As the bastion of Olympic sport (and arguably of athletic bodies), track and field athletics may contribute to the changing picture of women's bodies in sport and pose a challenge to orthodox femininity where muscles are actively developed and displayed by female athletes.

A second point for discussion concerns self-relatedness and the limitations of being bodies. The identity construction of this group takes a markedly physical form. The athletes in this study both desire and use the gaze of others when it concerns their muscularity, along with their own self-scrutiny, to construct and confirm their identities and their bodies as engaged in a process of transformation and development towards an ideal. This is a similar picture to the one observed by Brown (1999) in relation to bodybuilders, who became acutely aware that they are their bodies. Like the bodybuilders in Brown's (1999) study the athletes exude high levels of bodily control mediated through appearance pursuits, where the physical self is almost entirely constitutive of a self-identity formed around the notion of young sculpted athletic bodies. Accordingly, the limitations of being a body present concern. As Shilling (2012) notes all bodies' age and decay. The decline of the body with age presents an 
imminent threat to this group who structure appearances and actions on the criteria of youth and lean muscle which is increasingly difficult to attain and maintain. In this respect, body options for the future may be limited. Unlike body builders however, the body of the good track athlete is ultimately judged on an objective measure of performance, and not on appearance. The paradox, for these female track athletes, seems to lay less in balancing femininity with muscularity (as noted in previous studies, e.g. Krane et al, 2004) and more in their own (often confused) understanding of 'what they do' as being about performance or appearance. In this sense, we get an insight into the everyday lives of these females who are engaged in body project (Shilling, 2012) where the primary goal of performance of appearance is conflated for most.

A final point for discussion concerns the action problems of control and other-relatedness, shared by the disciplined and mirroring bodies, and social context. The athletes struggled with a change in social context, specifically when changing from training places (the gym and the indoor track) to social places (the coffee shop). Social context is crucial to Frank's (1991: 48) theory of the body where the body's own problems of its embodiment are always within a social context:

'Bodies alone have 'tasks'. Social systems may provide the context in which these tasks are defined, enacted, and evaluated, but social systems themselves have no 'tasks'. The theoretical problem is to show how social systems are built up from the tasks of bodies, which then allows us to understand how bodies can experience their tasks as imposed by a system.

The social system of the coffee shop does not align well with the tasks of the disciplined and mirroring bodies produced with ease in training contexts illuminating felt shifts in embodiment. For example, in accordance with Birke (in Shilling, 2012: xii), Esther's "uncomfortable situation" conveys an 'active response to change and contingency' in the coffee shop, where she (often subconsciously) monitors shifts in external environments and reacts to incoming stimuli on a level that is felt internally, in organs and tissues, much like Paradis' (2012) analogy of feeling like a 'fish out of water'. Findings illustrate how the body centrally matters for social interactions within social space.

Accordingly, these shifts might form a focal point for some of the concerns we have with such 'typical' or 'ideal' usage structured around control. For example, the ability to feel at ease in social places other than athletic ones. The medium of control has been raised as a concern for the highly disciplined sporting body elsewhere. We echo thoughts which focus upon the sporting body's crisis in a loss of control should it suffer career ending circumstances, for example serious illness or injury (Sparkes, 1998; Sparkes, 2004; Stewart et al, 2011).The lack of meaningful narrative resources to make sense of such losses are well documented in these studies and include detriments to mental health and even suicide attempts. This body of work focuses primarily upon Frank's (1991) disciplined body in male athletes and there is no work to date which explores the combination of high levels of mirroring and disciplined body usage in female athletes. Therefore this study raises concerns where female athletes, whose appearance matters, engage in highly mirroring and disciplined 
body usage for the most part of their daily lives mediated through high levels of control. These bodies in combination require further exploration in general and particularly in sports where the aesthetic is primary. Finally, concerns with a monadic way of relating to others might also extend to the personal and social development of the athletes. Giddens' (1991) echoes concerns about the ability of monadic bodies to relate sensuality to communication in dyadic relationships with others. This may be particularly so outside of sporting contexts.

To this end the study seeks to 'keep pace' with 'social and cultural trends that have made bodies increasingly contested components of social control, self-identity and individual action' (Shilling, 2012: ix) documenting a move towards understanding the athletic-ideal for females. Other emerging practices which adhere to similar ideals ought to be explored in a similar way (e.g. crossfit) to contribute to a fuller picture of the how the parameters of muscle and women's bodies are changing over time. We have posited Frank's (1995) typology as a useful intellectual tool to identify and discuss moments where the specific styles of 'ideal' bodies emerge in a female track athletic culture. Frank's theory of body usage allows us to understand how the body and its materiality matters, in the constant solving of problems for itself, in various social conditions and places. Limitations then may be found in the transitory focus of a group of white middle class female athletes. In this sense our research may seem to neglect 'race'/ethnicity and we recognise that this is something which requires further exploration. For example, the extent to which no 'upper limit' of muscularity was expressed may be intricately related their understanding of themselves as white and their relationship to hegemonic femininity (as opposed to how black athletes relate to these ideas for example). This said in the cultural context of track and field athletics we recognise that gender has important intersections with other social categories such as 'race'/ethnicity, social class and age, and that not all female track athletes will experience their embodiment in the same way. The experiences of black and other ethnic minority athletes will contribute valuable insight and therefore future work should build upon this study to explore muscularity and embodiment across intersections of gendered experience in athletics culture over time.

\section{Acknowledgments}

We would like to thank Gareth Wiltshire and Dominic Malcolm for their advice and attention given to reading earlier versions of this article.

\section{Notes}

\footnotetext{
${ }^{1}$ British heptathlete Jessica Ennis-Hill became not only the poster girl of athletics but of the London 2012 Olympic Games, reaping the benefits of positive media attention, fan adoration and sponsorship accordingly (Krane et al, 2004). She was chosen over other female athletes, including defending Olympic champion and world champion Christine Ohuruogu, a visibly more muscular 400m specialist who may not fit the cultural ideal as well as Ennis-Hill.
}

\footnotetext{
${ }^{2}$ We consider the possibility, having reflected on researcher diary notes, that the differing researcher positioning in terms of sexual orientation combined with the close personal
} 
relationships EP has with the group, opens up a space for deeper reflection and expression of desire for other women's bodies to be discussed more freely.

${ }^{3}$ One which aligns to the sub-cultural female athlete-ideal so often represented by sportswear company Nike, for example: tight fitting lycra (either leggings or 'hot pant' style shorts), crop tops or vests (usually of the female clothing line from selected brand, appropriate colours) and not far from the ideal Nike models which the group often refer to as an ideal image to mirror. The overall appearance of female track and field athletes is one of 'athletic, compact and neat' in appearance (which includes hair).

\section{References}

Allen-Collinson J (2009) Sporting Embodiment: Sports Studies and the (continuing) Promise of Phenomenology. Qualitative Research in Sport and Exercise 1(3): 279-296.

Allen-Collinson J and Hockey J (2007) Grasping the phenomenology of sporting bodies. International Review for the Sociology of Sport 42 (2):115-131.

Anderson L (2006) Analytic autoethnography. Journal of contemporary ethnography 35(4): 373-395.

Brewer JD (2000) Ethnography. Berkshire: Open University Press.

Brown D (1999) Male Bodybuilders and the Social Meaning of Muscle. Auto/Biography 1(2): 83-90.

Bruner EM (1997) Ethnography as narrative. In: Hinchman L and Hinchman S (eds) Memory, Identity, Community. The Idea of Narrative in the Human Sciences. New York: State University of New York Press, pp.264-281.

Bruner EM (1997) Ethnography as narrative. In: Hinchman L and Hinchman S (eds) Memory, Identity, Community: The Idea of Narrative in the Human Sciences. New York: State University of New York Press, pp.264-281.

Cole CL (1993) Resisting the canon: Feminist Cultural Studies, sport, and technologies of the body. Journal of Sport and Social Issues 17: 77-97.

Connell RW (2005) Masculinities. Cambridge: Polity Press.

Cox B and Thompson S (2000) Multiple bodies: Sportswomen, soccer and sexuality. International Review for the Sociology of Sport 35(1): 5-20.

Crossley N (2006) In the gym: Motives, meaning and moral careers. Body and Society 12(3): 23-50.

Curry TJ (1991) Fraternal bonding in the locker room: A profeminist analysis of talk about competition and women. Sociology of Sport Journal 8: 119-135. 
Davies CA (2002) Reflexive Ethnography. A Guide to Researching Selves and Others. London: Routledge.

Dworkin S (2001) 'Holding back': Negotiating a glass ceiling on women’s muscular strength. Sociological Perspective 44(3): 333-350.

Edwards J (1988) Political Cartoons in the Presidential Campaign: Image Metaphor and Narrative. NewYork: Garland.

Frank AW (1991) For a sociology of the body: An analytical review. In Featherstone M, Hepworth M and Turner BS (eds.) The Body: Social Processes and Cultural Theory. London: Sage, pp.36-102.

Frank AW (1995) The Wounded Storyteller. Body, Illness and Ethics. Chicago: University of Chicago Press.

George M (2005) Making sense of muscle: The body experiences of collegiate women athletes. Sociological Inquiry 75: 315-345.

Giddens A (1991) Modernity and Self Identity: Self and Society in the Late Modern age. Stanford CA: Stanford University Press.

Hall M (1996) Feminism and sporting bodies: Essays on theory and practice. Champaign, IL: Human Kinetics.

Hargreaves J (1993) Sporting females: Critical issues in the history and sociology of women's sports. New York: Routledge.

Harper D (2002) Talking about pictures: A case for photo elicitation. Visual Studies 17(1): 13-25.

Harrison B (2004) Photographic visions and narrative inquiry. In Bamberg M and Andrews M (eds.) Considering counter-narratives: Narrating, resisting and making sense. Amsterdam: John Benjamins Publishing Company pp.113-136.

Homan K (2010) Athletic-ideal and thin-ideal internalization as prospective predictors of body disatisfaction, dieting and compulsive exercise. Body Image 7: 240-245.

Kirmayer LJ (1992) The body's insistence on meaning: Metaphor as presentation and representation in illness experience. Medical Anthropology Quarterly 4: 323-346.

Krane V, Choi YL, Baird SM, Aimar, CM and Kauer, KJ (2004) Living the Paradox: Female Athletes Negotiate Femininity and Muscularity. Sex Roles 50(5-6): 315-329.

Markula P (1995) Firm but shapely, fit but sexy, strong but thin: The postmodern aerobicizing female bodies. Sport in Society Journal 12(4): 424-453. 
Menneson C (2000) 'Hard' women and 'soft' women. The social construction of identities among female boxers. International Review for the Sociology of Sport 35(1): 21-33.

Mosewich AD, Vangool AB, Kowalasi KC and McHugh T-LF (2009) Exploring women track and field athletes' meanings of muscularity. Journal of Applied Sport Psychology 21(1): 99-115.

Paradis E (2012) Boxers, Briefs or Bras? Bodies, Gender and Change in the Boxing Gym. Body and Society 18(2): 82-109.

Roussel P and Griffet J (2009) The path chosen by female bodybuilders: A tentative interpretation. Sociology of Sport Journal 17(2): 130-150.

Roussel P, Griffet J and Duret P (2003) The decline of female body building in France. Sociology of Sport Journal 20(1): 40-59.

Shilling C (2012) The Body and Social Theory ( $3^{\text {rd }}$ edition). London: Sage.

Soja E (1996) Thirdspace: journeys to Los Angeles and other real and imagined places. Oxford: Wiley-Blackwell.

Sparkes AC (1998) Athletic identity: An Achilles' Heel to the survival of self. Qualitative Health Research 8(5): 644-664.

Sparkes AC (2004) Bodies, narratives, selves and autobiography: The example of Lance Armstrong. Journal of Sport and Social Issues 28(4): 397-428.

Sparkes AC (2009) Ethnography and the senses: Challenges and possibilities. Qualitative Research in Sport and Exercise 1(1) 21-35.

Sparkes AC, Batey J and Brown D (2005) The muscled self and its aftermath: A life history study of an elite, black, male bodybuilder. Auto/Biography 13: 131-160.

Stewart C, Smith B and Sparkes A (2011) Sporting autobiographies of illness and the role of metaphor. Sport in Society 15(5): 581-597.

Theberge N (2000) Higher goals: Women's ice hockey and the politics of gender. New York: State University of New York Press.

Wesley JK (2001) Negotiating gender: Bodybuilding and natural/unnatural continuum. Sociology of Sport Journal 18(2): 162.

Woodward, K. (2011) The short guide to gender. Bristol: The Policy Press. 


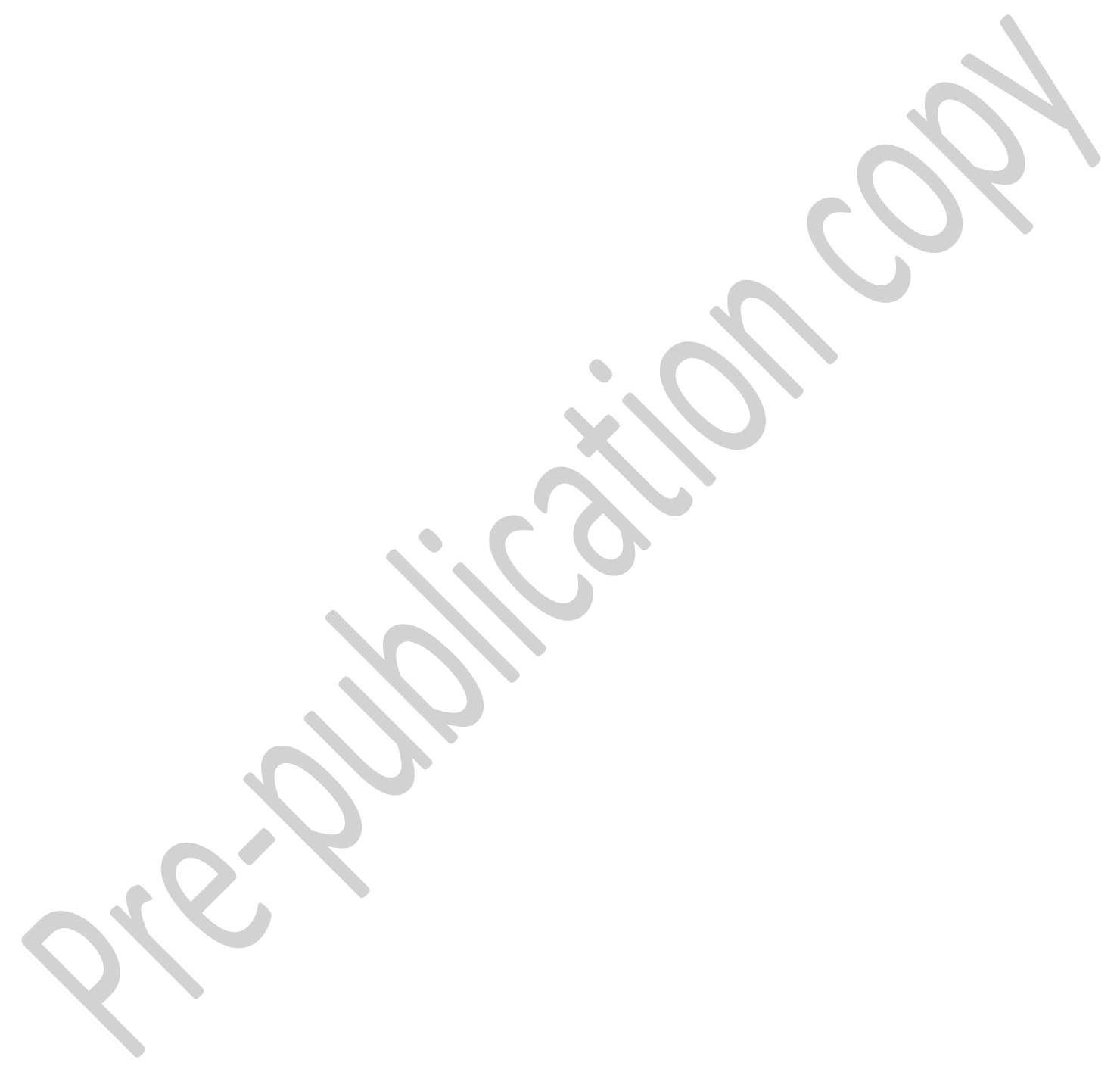

